\title{
Virtual Screening of Fargesin Analogs as Candidates as Inhibitors of Aedes aegypti Sterol Carrier Protein
}

\author{
Sammya Yasmin EM De Lima', Anderson Roberto Nery Da Silva', Cíntia Emi Yanaguibashi Leal' \\ Edilson B De Alencar Filho ${ }^{1,2,3,{ }^{*}}$
}

\section{Sammya Yasmin EM De Lima ${ }^{1}$, Anderson Roberto Nery Da Silva', Cíntia Emi Yanaguibashi Leal', Edilson B De Alencar Filho ${ }^{1,2,3, *}$}

'Department of Pharmaceutical Sciences, Federal University of Vale do São Franciso Campus Petrolina-Centro, BRAZIL.

2Post Graduation Program in Biosciences, University of Vale do São Franciso Campus Petrolina-Centro, BRAZIL. ${ }^{3}$ Post Graduation Program in Health and Biological Sciences, University of Vale do São Franciso Campus PetrolinaCentro, BRAZIL.

Correspondence

\section{Prof. Edilson B de Alencar Filho}

1 Department of Pharmaceutical Sciences, Federal University of Vale do São Franciso Campus Petrolina-Centro, BRAZIL.

${ }^{2}$ Post Graduation Program in Biosciences, University of Vale do São Franciso Campus Petrolina-Centro, BRAZIL.

${ }^{3}$ Post Graduation Program in Health and Biological Sciences, University of Vale do São Franciso Campus Petrolina-Centro, BRAZIL.

Email id: edilson.beserra@univasf.edu.br

History

- Submission Date: 08-10-2021;

- Review completed: 26-10-2021;

- Accepted Date: 21-12-2021

DOI : $10.5530 /$ pres. 14.1 .3

Article Available online

http://www.phcogres.com

Copyright

(C) 2022 Phcog.Net. This is an openaccess article distributed under the terms of the Creative Commons Attribution 4.0 International license.

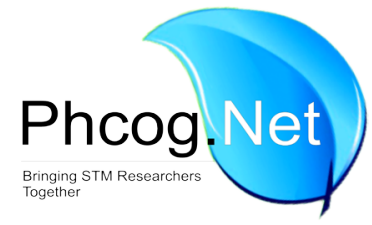

\begin{abstract}
Background: Fargesin is a natural product that was identified by our group in previous work as a potent ligand of the sterol-binding protein-2 (SCP-2) of Aedes aegypti, the mosquito that which causes the propagation of diseases such as zika, dengue, Chikungunya, and yellow fever. Objectives: propose structural analogs synthetically accessible to be evaluated by molecular docking, preserving the fargesin privileged group's benzodioxol and methoxybenzene, with different spacer groups. Materials and Methods: Structures were obtained in PubChem $®$ and SciFinder ${ }^{\circledR}$ databases, in .sdf format. Chemical structures were converted to pdbqt format by the OpenBabel GUI® program. Macromolecular target SCP-2 (PDB ID: 1PZ4) was extracted from the RSCB-PDB® platform, and molecular docking was performed using the Autodock vina program. The target binding site was defined based on the crystallographic ligand (palmitic acid), with grid box dimensions $16 \times 12 \times 18 \AA$. Results: The database built had 1231 molecules, with molecules that had binding energy lower than $-6.9 \mathrm{Kcal} / \mathrm{mol}$ considered valid results. Thus, of the 447 molecules that were most favorable, the best results were for the compounds: $188(-12.6 \mathrm{Kcal} / \mathrm{mol}), 350(-12.6 \mathrm{Kcal} / \mathrm{mol}), 351$ $(-12.3 \mathrm{Kcal} / \mathrm{mol}), 773(-12.3 \mathrm{Kcal} / \mathrm{mol})$ and $831(-12.1 \mathrm{Kcal} / \mathrm{mol})$. Conclusion: The results of this paper showed that isoflavonoids synthetically acessible are promising candidates for larvicidal agents, which corroborates with other published data from our research group and encourages experimental investigations.

Key words: Aedes aegypti, Fargesin, Isoflavonoids, Molecular docking, Synthetic Analogs.
\end{abstract}

\section{INTRODUCTION}

Every year more than one million people die from vector-borne diseases, of which mosquito-borne diseases represent a significant proportion. ${ }^{[1]}$ One of the most important vectors that transmit diseases is the Aedes aegypti mosquito, which causes the propagation of diseases such as zika, dengue, Chikungunya, and yellow fever. ${ }^{[2]}$ Given that a single mosquito is the primary vector of multiple human diseases, the development of new strategies for mosquito control is crucial. ${ }^{[3]}$ The most common way to combat the mosquito is with insecticides. However, they are toxic to humans and the environment. ${ }^{[4]}$ Another concern about chemical agents is the increasing resistance of the mosquito vector. ${ }^{[5]}$ In this context, natural products, especially secondary metabolites, have emerged as agents that are generally safer for humans and that generate fewer residues in the environment. ${ }^{[6]}$ In a previous work of our laboratory, we investigated the affinity of 248 secondary metabolites of plants from the Caatinga Biome as potential ligands of Steroid Carrier Protein-2 (SCP-2) from Aedes aegypti. Among the highlighted compounds, we identified Fargesin, ${ }^{[7]}$ a compound with multiple pharmacological activities, including attenuation of oxidative stress and anti-inflammatory activity in monocytic cells, ${ }^{[8]}$ increase in basal glucose uptake by translocating Insulin-Sensitive Glucose Transporter (GLUT4), ${ }^{[9]}$ antihypertensive activity, ${ }^{[10]}$ inhibition of melanin synthesis, ${ }^{[11]}$ and antitrypanosomal activity. ${ }^{\text {pere }}$

Fargesin belongs to the class of lignans (also known as neolignans). Lignans are found in plants of Aristolochia genus popularly known as "mil-homens" or "jarrinha"; and in plants of Magnolia genus commonly known as "pinha - do - brejo" or "baguaçu". ${ }^{[12-14]}$ This metabolite is derived from the oxidative coupling of two phenylpropanoid units and propenylphenols (isoeugenol and coniferyl alcohol), with regiospecific and diastereospecific control. [15] Different monomeric precursors generate new subgroups of lignans by cyclization or modifications in the carbon skeleton, resulting in a wide variety of substances. ${ }^{[13]}$ Within these subclasses, Fargesin is classified as furanic lignan, characterized by a benzodioxol ring, bonded to a linker structure of furanoid rings bonded to dimethoxybenzene, ${ }^{[11]}$ (Figure 1).
Cite this article: Lima SYEMD, Silva ARND, Leal CEY, Filho EBDA. Virtual Screening of Fargesin Analogs as Candidates as Inhibitors of Aedes aegypti Sterol Carrier Protein. Pharmacog Res. 2022;14(1):12-8. 


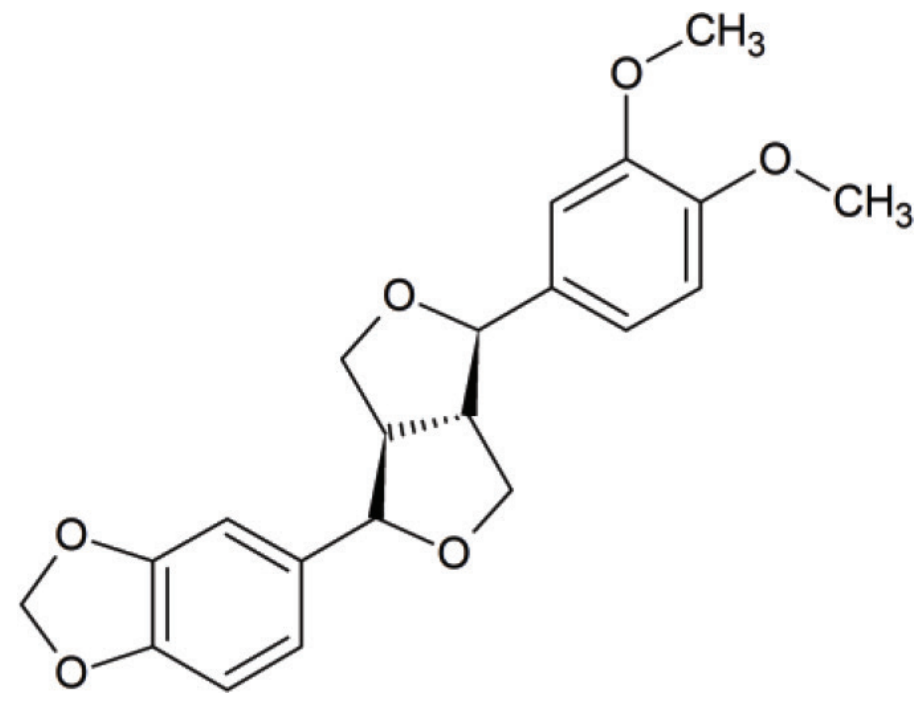

Figure 1: Fargesin.

Given the already known biological characteristics of Fargesin, as well as its potential previously identified by our group, this study aimed to perform a virtual screening study using Molecular Docking (MD), applied to a set of 1231 molecules designed for the synthesis of Fargesin analogs. In these molecular architectures, two privileged structures of the original lignan were preserved, the benzodioxol and dimethoxybenzene systems, replacing only the intermediate furan ring system by linker structures, such as those from the Morita Baylis-Hillman reaction, hydrazones, as well as their retroisosteres. Subsequently, the molecules were tested via docking against the same Aedes aegypti sterol-2-binding protein aiming to identify more promising compounds for future synthesis and bench testing.

\section{MATERIALS AND METHODS}

\section{Design and Selection of Analogs}

Fargesin analogs with designed linkers were searched using PubChem ${ }^{\circ}$ and SciFinder ${ }^{\oplus}$ databanks. The structures were found using a search tool, where it was possible to draw the molecule. The proposed compounds were designed to keep the privileged structures of Fargesin, with spacer structures such as Morita Baylis-Hillman (ester, nitrile), hydrazones, and retroisosteres.

The selection criteria were molecules that showed similarity to the proposed molecule. A total of 1231 compounds were selected for Molecular Docking (MD). Then, the 3D molecules were downloaded in SDF file extension and further converted by Open Babel GUI program, ${ }^{[16]}$ to the mol file extension for edition in ChemSketch ${ }^{\circledR}$ program (ACD/ChemSketch, version 2012, Advanced Chemistry Development, Inc., Toronto, ON, Canada, www.acdlabs.com, 2021); and finally, converted directly by Open Babel GUI ${ }^{\circledast}$ software, ${ }^{[16]}$ to pdbqt file.

\section{Macromolecular target (1PZ4)}

The macromolecular target for the evaluation of the larvicidal potential was the Aedes aegypti sterol-binding protein, which crystallographic structure can be found in the Protein Data Bank database, registered under PDB code: 1PZ4. This protein was obtained complexed with its native ligand, a fatty acid known as palmitic acid. After that, the protein was modeled and prepared in Autodock software to add Gastaiger charges and polar hydrogens, which are absent in crystallographic structures, and saved in a pdbqt file for MD.

\section{Molecular docking}

Before MD, redocking (RDK) was conducted with the co-crystallographic ligand to determine the accuracy of the docking procedure. All calculations were performed with the Autodock ${ }^{\otimes}$ Vina module. ${ }^{[17]}$ The grid box dimensions chosen were $16 \times 12 \times 18 \AA$, centered on the ligand and with the standard spacing of $1 \AA$ between the internal grid points. The same parameters previously described were applied to the docking of the 1231 synthetic analogs of Fargesin. The 3D images and diagrams were generated with the free module of the Schrödinger Maestro software (Maestro, Schrödinger, LLC, New York, NY, 2021).

\section{RESULTS AND DISCUSSION}

After searches in PubChem ${ }^{\star}$ and SciFinder ${ }^{\circledR}, 1231$ Fargesin analogs were extracted and tested on the Aedes aegypti sterol-binding protein. Table 1 shows the compounds that had energy lower than $-6.9 \mathrm{Kcal} / \mathrm{mol}$, totaling 447 favorable compounds. Table 1 also shows the redocking (RDK) result. It was found that molecules $188,350,351,773$, and 831 had an excellent interaction with the protein, achieving binding energy almost twice as stable as RDK and with very slight energy variation between each other. In other words, binding energies had close values. This study aims to discuss the best five molecules.

Of the compounds that stabilized the protein, with energies from -12 $\mathrm{kcal} / \mathrm{mol}$, three are synthetic isoflavonoids, like molecules 188, named (2-methyl-4-oxo-3-phenylchromen-7-yl)-1,3- benzodioxol-5-carboxylate; 350, named 3-(4-methoxyphenoxyl)-2-methyl-4-oxochromen-7-yl] 1,3-benzodioxol-5-carboxylate; and 351, named [3-(4-methoxyphenyl)4-oxochromen-7-yl] 1,3-benzodioxol-5-carboxylate. All molecules are analogous to each other. The structures of these compounds are formed by a benzodioxol ring linked by the ester function to the chromone ring, linked to the methoxybenzene ring. For molecule 188, only the benzene ring has no substituent (Figure 2).

The isoflavonoids contain a methoxyl group at the "para" position of the ring B. Compounds 188, 350, and 351 are derived from 7-hydroxy4-methoxyflavone, a naturally occurring isoflavonoid known as Formononetin, found in legumes. ${ }^{[18]}$ The molecules are obtained through the alcoholysis reaction, where Formononetin participates as a reagent and thereby forms analogous and biosynthetic molecules. Formononentin derivatives are widely studied because they can reverse multidrug resistance by inhibiting the efflux pump. Furthermore, also have antihypertensive, anti-parasitic, ${ }^{[18]}$ breast and prostate cancer, and antioxidant activity. ${ }^{[19]}$

Figure 3 shows molecules 188, 350, 351 and their interactions. Note that in all three molecules, the carboxylate group, which binds the benzodioxol group to the chromone ring, interacts with the same amino acids as the native ligand in the main chain, which are Valine 26 (Val 26), Glycine 25 (Gln 25), and Arginine 24 (Arg 24). These interactions occur through hydrogen bonds, represented by the pink arrow in the $2 \mathrm{D}$ diagram. Besides the hydrogen bond with these amino acid residues, there is another hydrogen bond between Arg 15 and the oxygen of benzodioxol.

Molecule 773, named 3-[(E)-3-(1,3-benzodioxol-5-yl)prop-2-enoyl]7-[(3-methoxyphenyl)methoxy]chromen-2-one belongs to the class of polyoxygenated cinnamoylcoumarins. This class is derived from curcumin, which is obtained through the synthetic route with hydroxysalicylaldehyde as a reagent. ${ }^{[20]}$ Like other molecules discussed, cinnamoylcoumarins have biological importance. Resembling curcumin, they possess antioxidant properties, as well as cytotoxic and anticancer effects. ${ }^{[20]}$

Structurally, molecule 773 has a methoxyl at the "ortho" position of ring A. It can also be observed that between the benzodioxol ring and the 
Table 1: Binding energy of compounds for larvicidal activity in ascending order.

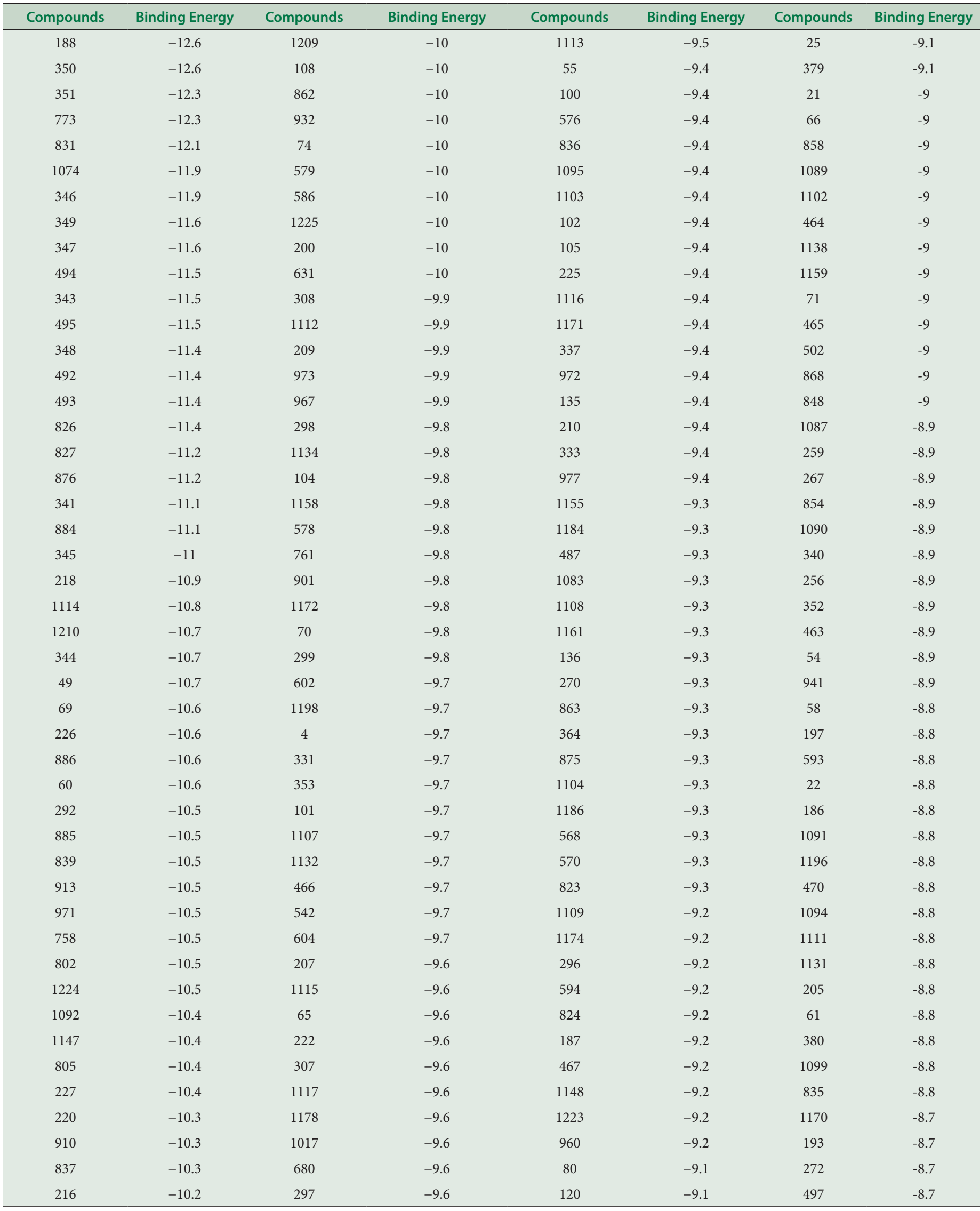

continued... 
Table 1: Binding energy of compounds for larvicidal activity in ascending order.

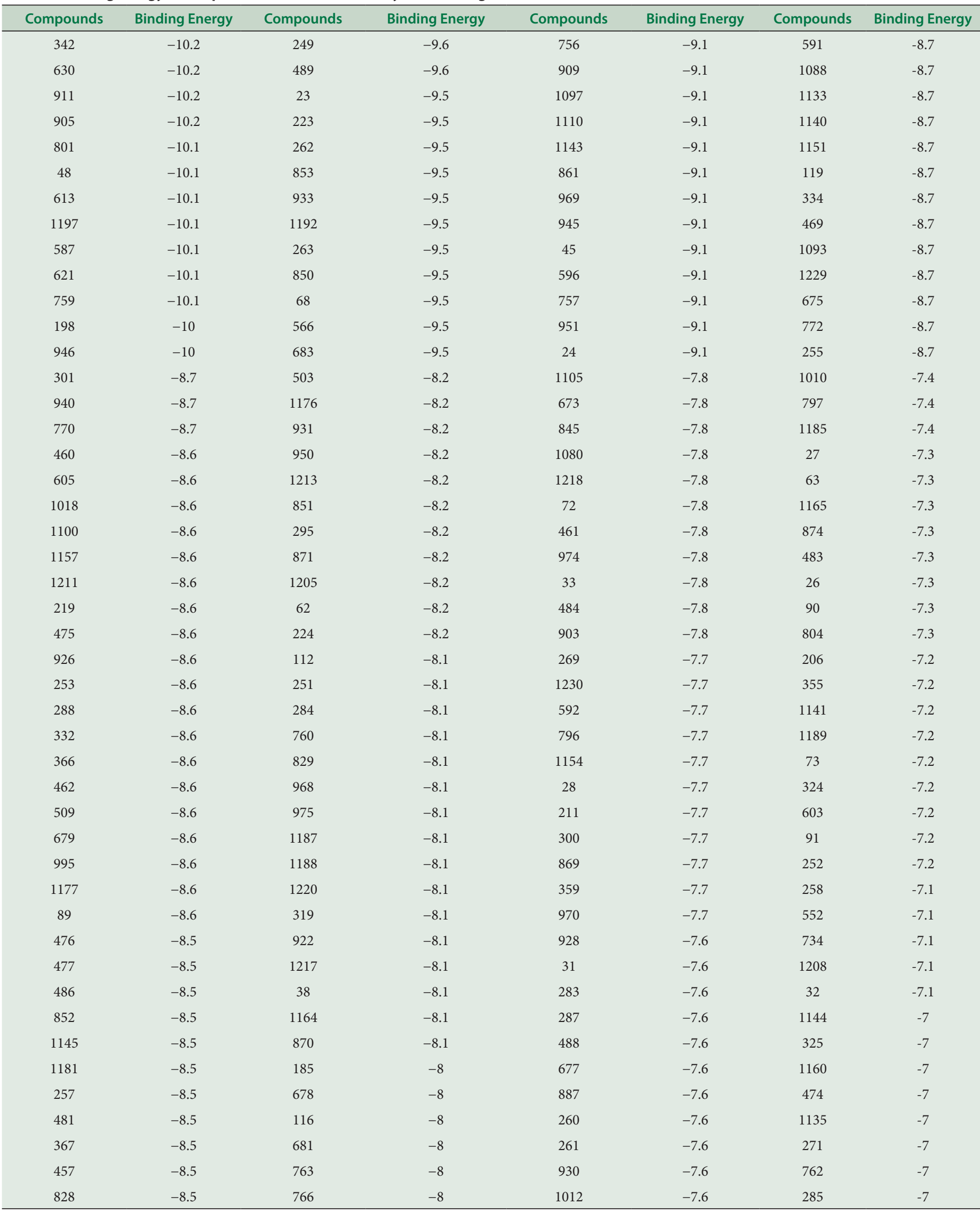


Table 1: Binding energy of compounds for larvicidal activity in ascending order.

\begin{tabular}{|c|c|c|c|c|c|c|c|}
\hline Compounds & Binding Energy & Compounds & Binding Energy & Compounds & Binding Energy & Compounds & Binding Energy \\
\hline 840 & -8.5 & 956 & -8 & 378 & -7.6 & 739 & -7 \\
\hline 976 & -8.5 & 291 & -8 & 1146 & -7.6 & $\mathrm{RDK}$ & -6.9 \\
\hline 328 & -8.4 & 1204 & -8 & 67 & -7.6 & & \\
\hline 29 & -8.4 & 290 & -8 & 268 & -7.6 & & \\
\hline 920 & -8.4 & 316 & -7.9 & 1190 & -7.6 & & \\
\hline 264 & -8.4 & 814 & -7.9 & 1126 & -7.5 & & \\
\hline 265 & -8.4 & 856 & -7.9 & 228 & -7.5 & & \\
\hline 480 & -8.4 & 999 & -7.9 & 479 & -7.5 & & \\
\hline 849 & -8.3 & 86 & -7.9 & 952 & -7.5 & & \\
\hline 1098 & -8.3 & 590 & -7.9 & 1231 & -7.5 & & \\
\hline 322 & -8.3 & 64 & -7.9 & 36 & -7.5 & & \\
\hline 1180 & -8.3 & 302 & -7.9 & 966 & -7.5 & & \\
\hline 674 & -8.3 & 599 & -7.9 & 670 & -7.5 & & \\
\hline 82 & -8.3 & 859 & -7.9 & 245 & -7.4 & & \\
\hline 458 & -8.3 & 1169 & -7.9 & 182 & -7.4 & & \\
\hline 944 & -8.3 & 1221 & -7.9 & 273 & -7.4 & & \\
\hline 459 & -8.3 & 908 & -7.9 & 309 & -7.4 & & \\
\hline
\end{tabular}

Binding energies are represented in $\mathrm{kcal} / \mathrm{mol}$. RDK: reocking.
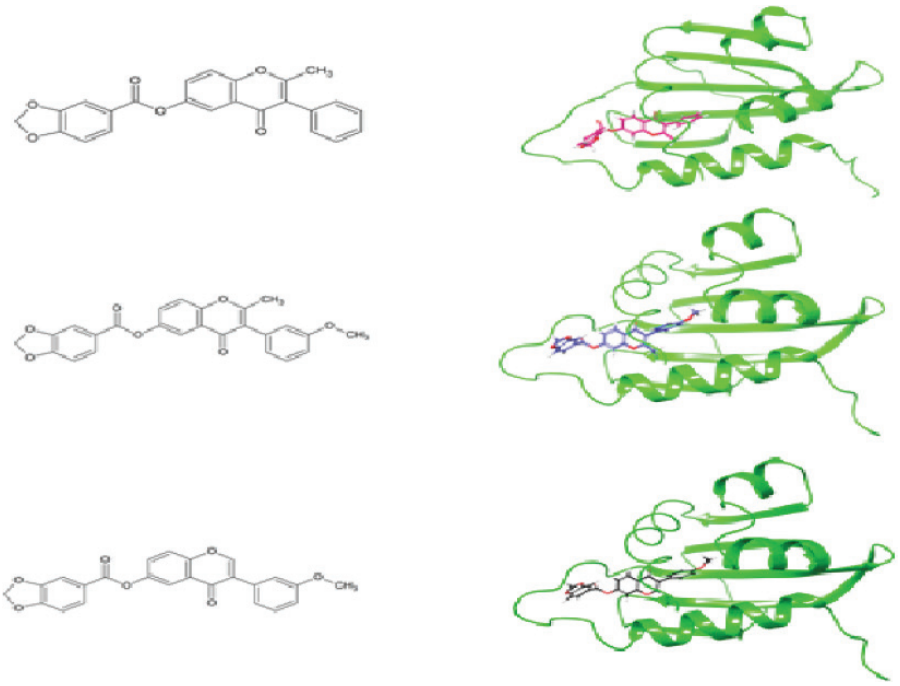

Figure 2: Compounds 188 (A), 350 (B) and 351 (C) in 2D and complexed with 1 PZ4.
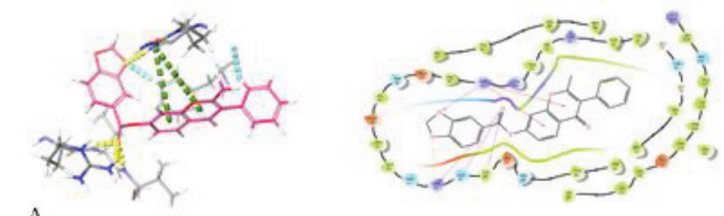

A
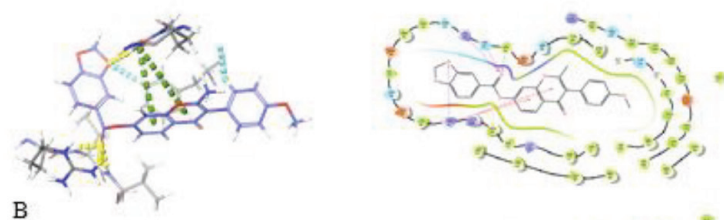

$\mathrm{C}$
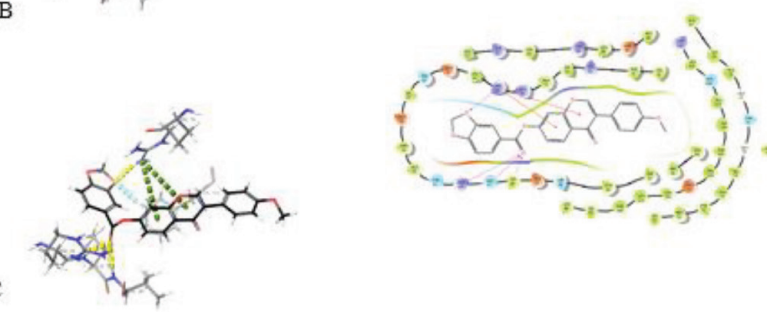

Figure 3: Molecules 188 (A), 350 (B) e 351 (C) interacting with amino acid residues in the protein. In green pi-cation-type bond, in yellow hydrogen bond, in cyan aromatic. 


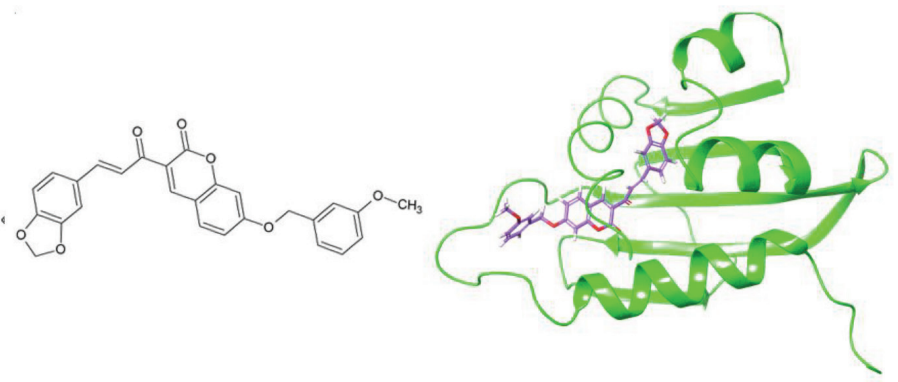

Figure 4: Molecule 773 in 2D (left) and complexed to 1PZ4 (right).
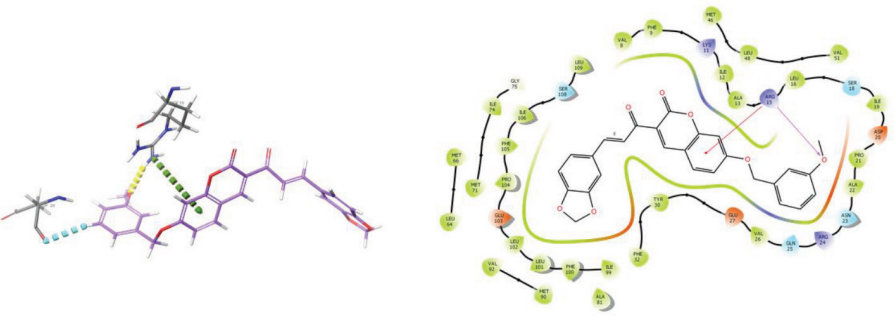

Figure 5: Molecule 773 (left) interacts with the amino acid Asp 20 through aromatic hydrogen bond (cyan), as well as interacting with Arg 15 by hydrogen bond (in yellow) and pi-cation-type bond (green dots). Diagram of interactions is represented on the right. The red arrows represent pi - cation interactions.

chromene ring, it has an E-type (trans) stereoisomer and a carbonyl; and a methoxybenzene bonded to the chromene ring via ethyl ether (Figure 4).

Unlike the other molecules, compound 773 had the methoxybenzene group toward the external opening of the hydrophobic pocket, while in the other molecules the benzodioxol group was directed toward the internal region (Figure 4). As a consequence, this compound did not interact with the main chain amino acids Val 26, Gln 25, and Arg 24, only with the side chain residue Arg 15 via hydrogen bonding with the methyl ether of methoxybenzene, represented by the pink arrow in Figure 4 on the right and yellow dotted on the left. Other interactions were also observed, such as pi-cation type interaction and charge type bonding with Arg 15 (Figure 5). On the left of Figure 5, it is also possible to see an aromatic hydrogen bonding with Aspartate 20 (Asp 20).

Molecule 831, named:4-[6-[2-[2-[1-(1,3-benzodioxol-5-yl)cyclopropyl]2-oxoethyl]pyridin-3-yl]benzoic acid, is an organic acid structurally different from 773 and molecules derived from Formononetin. It has in its structure a pyridine linked to a carbonyl and a cyclopropane (Figure 6). This molecule is widely studied, mainly because of its potential to modulate ABC (ATP Binding Cassette), a protein that is responsible for multidrug resistance and is involved in diseases such as Alzheimer's, Amyotrophic Lateral Sclerosis, mucopolysaccharidosis. ${ }^{[21]}$

The benzyl carboxylic group was directed toward the external opening of the hydrophobic pocket, similar to compound 773. As a result, only one hydrogen bonding type interaction occurs between the main chain residue, Val 26, and the hydroxyl of the acid group. The non-covalent type interaction was the pi-pi stacking interaction with the phenylalanine residue 105 (Phe 105). Pi-pi stacking bond occurs between two aromatic rings, which can be parallel to each other, known as a sandwich (faceto-face) interaction, as seen in Figure 7 , side-to-side or Boda-face interaction. ${ }^{[22]}$

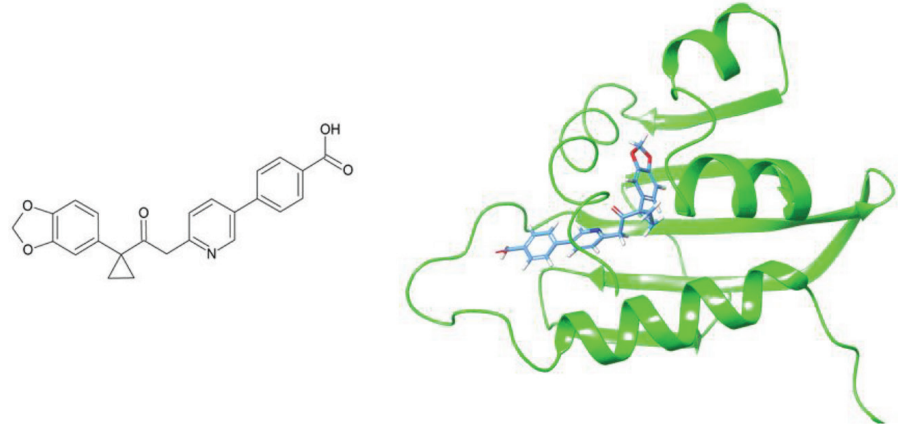

Figure 6: 2D representation of the 831 molecule (left), and the 3D representation coupled to 1 PZ4 (right).
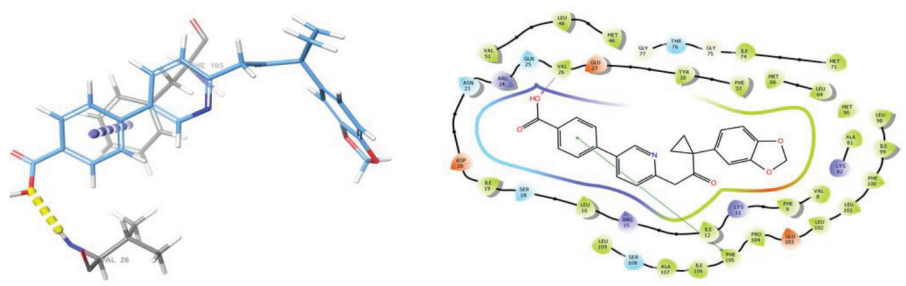

Figure 7: Molecule 831 (left) coupled to the protein, forming hydrogen bonds with Val 26 (yellow dots), and pi-pi stacking bonds (purple) with Phe 105 (right).

\section{CONCLUSION}

In this paper, we evidenciated natural product analogs with potencial against Aedes aegypti mosquitoes. Among the most stable compounds evaluated by dockings, the results emphasizes the isoflavonoid class as the best potential to interruption of the mosquito's life cicle. The synthetic obtention of these analogs has a simpler methodology and the reagents used are economically accessible, making them promising candidates for Sterol Carrier Protein-2 inhibition. Therefore, this work encourages the experimental evaluation of formononetin and analogs.

\section{ACKNOWLEDGEMENT}

The authors would like to thank the Coordination of Improvement of Higher Level Personnel (CAPES), National Council for Scientific and Technological Development (CNPq), and Foundation of Support to the Science and Technology of the State of Pernambuco (FACEPE) for the scholarship granted for the students.

\section{CONFLICT OF INTEREST}

The authors declare no conflict of interest.

\section{ABBREVIATIONS}

SCP-2: Steroid Carrier Protein-2; GLUT4: Insulin-Sensitive Glucose Transporter; MD: molecular docking; RDK: redocking.

\section{REFERENCES}

1. World Health Organization. A global brief on vector-borne diseases. World Heal Organ. 2014:1-56.

2. Mota MTde O, Terzian AC, Silva MLCR, Estofolete C, Nogueira ML. Mosquitotransmitted viruses - the great Brazilian challenge. Braz J Microbiol. 2016;47;Suppl 1:38-50. doi: 10.1016/j.bjm.2016.10.008, PMID 27818091. 
3. Powell JR. Mosquito-borne human viral diseases: why Aedes aegypti? Am $J$ Trop Med Hyg. 2018;98(6):1563-5. doi: 10.4269/ajtmh.17-0866, PMID 29557341.

4. Roman P. History, presence and perspective of using plant extracts as commercial botanical insecticides and farm products for protection against insects - a review. Plant Protect Sci. 2016;52(4):229-41. doi: 10.17221/31/2016PPS.

5. Dusfour I, Vontas J, David JP, Weetman D, Fonseca DM, Corbel V, et al. Management of insecticide resistance in the major Aedes vectors of arboviruses: advances and challenges. PLOS Negl Trop Dis. 2019;13(10):e0007615. doi: 10.1371/journal.pntd.0007615, PMID 31600206.

6. Silvério MRS, Espindola LS, Lopes NP, Vieira PC. Plant natural products for the control of Aedes aegypti: the main vector of important arboviruses. Molecules. 2020;25(15). doi: 10.3390/molecules25153484, PMID 32751878.

7. Mourão EDS, Carvalho TGCd, Lima SYEMd, Alencar Filho EBd. Identification of molecular scaffolds from Caatinga Brazilian biome with potential against Aedes aegypti by molecular docking and molecular dynamics simulations. J Mol Struct. 2021;1229. doi: 10.1016/j.molstruc.2020.129621.

8. Yue B, Ren YJ, Zhang JJ, Luo XP, Yu ZL, Ren GY, et al. Anti-inflammatory effects of fargesin on chemically induced inflammatory bowel disease in mice. Molecules. 2018;23(6):1-13. doi: 10.3390/molecules23061380, PMID 29880739

9. Lee YS, Cha BY, Choi SS, HaradaY, Choi BK, Yonezawa T, et al. Fargesin improves lipid and glucose metabolism in 3T3-L1 adipocytes and high-fat diet-induced obese mice. BioFactors. 2012;38(4):300-8. doi: 10.1002/biof.1022, PMID 22674784

10. Sha $S, X u$ D, Wang $Y, Z$ hao $W, L i X$. Antihypertensive effects of fargesin in vitro and in vivo via attenuating oxidative stress and promoting nitric oxide release. Can J Physiol Pharmacol. 2016;94(8):900-6. doi: 10.1139/cjpp-2015-0615, PMID 27409158

11. Fu T, Chai B, Shi Y, Dang $Y, Y e X$. Fargesin inhibits melanin synthesis in murine malignant and immortalized melanocytes by regulating PKA/CREB and P38/ MAPK signaling pathways. J Dermatol Sci. 2019;94(1):213-9. doi: 10.1016/j. jdermsci.2019.03.004, PMID 30956031.
12. Sartorelli P, Carvalho CS, Reimão JQ, Lorenzi H, Tempone AG. Antitrypanosomal activity of a diterpene and lignans isolated from Aristolochia cymbifera. Planta Med. 2010;76(13):1454-6. doi: 10.1055/s-0029-1240952, PMID 20301059.

13. Pereira M. Estudo fitoquímico de Aristolochia cordigera e síntese de lignanas com potencial antimalárico. Chemistry D [thesis], Chemistry institute. Araraquara, Brazil: Universidade Estadual Paulista; 2017.

14. Kassuya CAL, Cremoneze A, Barros LFL, Simas AS, Lapa Fda R, Mello-Silva R, et al. Antipyretic and anti-inflammatory properties of the ethanolic extract, dichloromethane fraction and costunolide from Magnolia ovata (Magnoliaceae). J Ethnopharmacol. 2009;124(3):369-76. doi: 10.1016/j.jep.2009.06.003, PMID 19524658.

15. Messiano GB, Vieira L, Machado MB, Lopes LMX, De Bortoli SA, ZukermanSchpector J. Evaluation of insecticidal activity of diterpenes and lignans from Aristolochia malmeana against Anticarsia Gemmatalis. J Agric Food Chem. 2008;56(8):2655-9. doi: 10.1021/jf703594z, PMID 18380460.

16. O'Boyle NM, Banck M, James CA, Morley C, Vandermeersch T, Hutchison GR Open babel: An open chemical toolbox. J Cheminform. 2011:3(33):33. doi: 10.1186/1758-2946-3-33, PMID 21982300.

17. Morris GM, Goodsell DS, Halliday RS, Huey R, Hart WE, Belew RK, et al. Automated docking using a Lamarckian genetic algorithm and an empirical binding free energy function. J Comput Chem. 1998;19(14):1639-62. doi 10.1002/(SICI)1096-987X(19981115)19:14<1639::AID-JCC10>3.0.CO;2-B.

18. Mutai $P$, Rigo GV, Tasca T. Synthesis and antigiardial activity of formononetin analogues. East and Central African. J Pharm Sci. 2016;16(1-3):22-7.

19. Bondarenko SP, Frasinyuk MS, Khilya VP. Synthesis of formononetin analogs. Chem Nat Compd. 2003;39(4):344-8. doi: 10.1023/B:CONC.0000003413.2380 8.2b.

20. Al Rihani SB, Darakjian LI, Deodhar M, Dow P, Turgeon J, Michaud V. Diseaseinduced modulation of drug transporters at the blood-brain barrier level. Int J Mol Sci. 2021;22(7):4-6

21. Cox JR. Teaching non-covalent interactions in the Biochemistry Curriculum through Molecular Visualization: the search for $\pi$ interactions. J Chem Educ. 2000;77(11):1424-8. doi: 10.1021/ed077p1424.

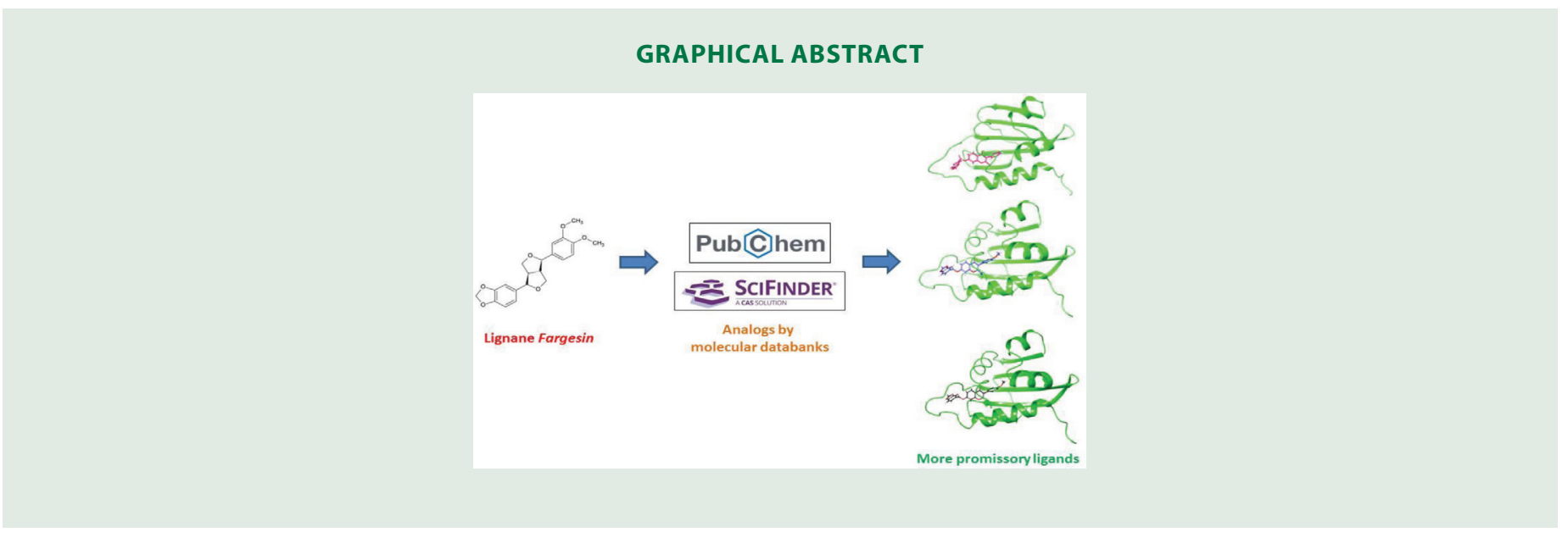

Cite this article: Lima SYEMD, Silva ARND, Leal CEY, Filho EBDA. Virtual Screening of Fargesin Analogs as Candidates as Inhibitors of Aedes aegypti Sterol Carrier Protein. Pharmacog Res. 2022;14(1):12-8. 